\title{
Editorial zur Wirbelsäulenarthroplastik (Non-Fusion - Techniken)
}

„Dekompression”, „Korrektur” und „Stabilisierung” waren bis vor etwa 10 Jahren die nahezu ausschließlichen und zentralen Operationskategorien in der Wirbelsäulenchirurgie. Die historische Entwicklung dieses Spezialgebietes war geprägt durch die operativen Therapiemöglichkeiten bei Deformitäten, Frakturen, Infektionen und Tumoren mit dem Ziel, stets o.a. Operationskonzepte in unterschiedlicher Ausprägung zu realisieren.

„Unerwünschte Nebenwirkungen” wie eine Versteifung mit ein- oder mehrsegmentalem Funktionsverlust, eine Nachbarsegment-Degeneration, ein iatrogenes Gewebstrauma oder operationsimmanente Komplikationen wurden aufgrund fehlender Alternativen billigend in Kauf genommen. Verbesserte Operationstechniken durch Anwendung der Prinzipien minimal-invasiver Chirurgie, optimierte optische Hilfsmittel wie Operationsmikroskop oder Endoskop sowie die Neuund Weiterentwicklung von Implantaten haben die operativen Therapieoptionen ebenso erweitert wie es die Fortschritte in der präoperativen und intraoperativen Bildgebung gewährleisten.

Die Erweiterung der Maßnahmen-Trias „Dekompression“, „Korrektur“ und „Stabilisierung“ auf das Spektrum degenerativer Erkrankungen war eine logische Folge. Die sinkende Akzeptanz für die mit lumbalen Fusionsverfahren assoziierten Nachteile hat gleichermaßen die Entwicklung sowohl schonender Zugangsverfahren wie auch rekonstruktiver, funktionserhaltender Techniken begünstigt.

Das vorliegende Themenheft befasst sich mit solchen Techniken. Trotz sehr unterschiedlicher, vorwiegend durch verschiedene Implantatsysteme getriggerter, Verfahren lassen sich gewisse Grundprinzipien zu Zugangs - und Behandlungsphilosophien darstellen. $\mathrm{Zu}-$ gangsstrategien zur Implantation von Bandscheibenendoprothesen an der Hals- und Lendenwirbelsäule stellen einen Fokus dieses Themenheftes dar. Es werden die gängigen, minimal-invasiven Operationstechniken einschließlich der Implantationstechniken für Kiel- und Non-Kiel-Endoprothesen wiedergegeben.

Sogenannte dynamische dorsale Pedikelschraubensysteme, deren Rolle bezüglich Indikation und klinischer Anwendung noch nicht endgültig definiert ist, werden ebenso präsentiert wie sogenannte interspinöse „Spacer”, deren Wirkprinzip auf einer indirekten Dekompression des Spinalkanals und einer Entlastung der Wirbelgelenke und Bandscheiben beruht und die durch die Möglichkeit der interspinösen Fixation auch als Alternative zu Pedikelschraubensystemen diskutiert werden.

Mit Ausnahme der künstlichen Bandscheiben, welche mittlerweile weltweit als Routine-Behandlungsoption akzeptiert sind, stellen alle anderen Verfahren innovative Ansätze dar, für die zwar zunehmend empirisch erhobene wissenschaftliche Daten akkumulieren, deren Rolle in der Routine-Anwendung allerdings bislang nicht als evidenz-basiert gelten kann.

Umso wichtiger ist es, die Details der in diesem Heft präsentierten Operationstechniken zu kennen und in der täglichen Anwendung umzusetzen.

H. Michael Mayer Andreas Korge 